\title{
Coherence Amplification in Off-Diagonal Density Matrix Elements
}

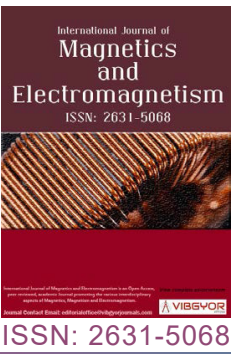

\section{Dennis J Sorce*}

Retired, Center for Magnetic Resonance Research, Minneapolis, USA

\begin{abstract}
We demonstrate coherence amplification in off-diagonal density matrix elements for a decoherence model for spin $1 / 2$ particles with applied radio-frequency pulse without relaxation. The method of coherence amplification developed may be of general utility in applications in NMR/MRI.
\end{abstract}

\section{Introduction}

The manifestation of coherence phenomena is a hallmark of quantum mechanics, differentiating it from classical phenomenon due to the property of superposition in quantum reality due to the linearity of the Schroedinger equation [1-4].

Methods of possible amplification of coherence as quantitated by the absolute value of off-diagonal density matrix elements, may be of use in applications such as quantum computing [5] and MRI tissue contrast $[6,7]$.

In the Letter, we develop a Possible Methodology of Coherence Enhancement. It is easily implemented and may have value in Venues of NMR/MRI [8].

\section{Formalism Development}

We base our Treatment on the Following Expression often used in Formalism of Coherence [1,9].

$$
\frac{d \hat{\rho}[t]}{d t}=-I\left[\hat{H}_{R F}[t], \hat{\rho}[t]\right]-\mathrm{T}\left[\tau_{d p h}\right]\left[\hat{H}_{R F}[t],\left[\hat{H}_{R F}[t], \hat{\rho}[t]\right]\right]
$$

Here, $\hat{\rho}[t]$ is the time-dependent Density Matrix. $\hat{H}_{R F}[t]$ is a Time Dependent Radio-Frequency Hamiltonian, defined as:

$$
\hat{H}_{R F}[t]=I_{x} w_{1}[t]+I_{z} \Delta w[t]
$$

$\hat{I}_{\alpha} \alpha=x, z$ are Spin $1 / 2$ Angular Momentum Operators [8]. The Radio-Frequency terms $w_{1}[t]$ and $\Delta w[t]$ are respectively, Amplitude and Frequency Offset Variable defined in Appendix I.

*Corresponding author: Dennis J Sorce, Retired, Center for Magnetic Resonance Research, Minneapolis, MN 55455, USA, Tel: 410-628-2461

Accepted: August 07, 2021; Published: August 09, 2021

Copyright: (c) 2021 Sorce DJ. This is an open-access article distributed under the terms of the Creative Commons Attribution License, which permits unrestricted use, distribution, and reproduction in any medium, provided the original author and source are credited.

Sorce. Int J Magnetics Electromagnetism 2021, 7:037

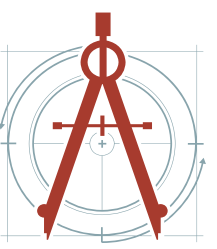


$\mathrm{T}\left[\tau_{d p h}\right]=\frac{k_{B} T}{h} \tau_{d p h}^{2}$

Here, $k_{B}$ is Boltzmann's Constant.

$h$ is Temperature in Kelvins.

$h$ is Planck's Constant Divided by $2 \pi$.

$\tau_{d p h}$ is a Time Constant Characterizing the Decoherence.

For the Specific Cases Treated here, to Explore a Model of Coherence Enhancement Effects, we specialize to the HS1 Adiabatic Pulse as detailed by Garwood, et al. [10] [see Appendix II].

If one substitutes Eq (2) into Eq (1), one obtains after "straightforward but tedious algebra":

$$
\begin{aligned}
& \frac{d \hat{\rho}[t]}{d t}=-I\left[\hat{H}_{R F}[t], \hat{\rho}[t]\right]+\mathrm{T}\left[\tau_{d p h}\right]\left(w_{1}^{2}[t] \hat{I}_{x} \hat{\rho}[t] \hat{I}_{x}+\Delta w^{2}[t] \hat{I}_{z} \hat{\rho}[t] \hat{I}_{z}\right. \\
& \left.-\frac{\hat{1}}{4} w_{e f f}^{2}[t] \hat{\rho}[t]+w_{1}[t] \Delta w[t]\left(\hat{I}_{x} \hat{\rho}[t] \hat{I}_{z}+\hat{I}_{z} \hat{\rho}[t] \hat{I}_{x}\right)\right)
\end{aligned}
$$

Where:

$$
w_{e f f}^{2}[t]=w^{2}{ }_{1}[t]+\Delta w^{2}[t]
$$

And:

$\hat{1}$ is the 2 by 2 Identity Matrix.

For Clarity and Completeness, we Explicitly Define the Following Terms:

$$
\hat{\rho}[t]=\left(\begin{array}{ll}
\rho_{11}[t] & \rho_{12}[t] \\
\rho_{21}[t] & \rho_{22}[t]
\end{array}\right)
$$

We adopt the Standard Definition of the Spin-1/2 Cartesian Spin Angular Momentum Operators

As:

$$
\begin{aligned}
& \hat{I}_{x}=\frac{1}{2} \sigma_{x,} \hat{I}_{y}=\frac{1}{2} \sigma_{y,} \hat{I}_{z}=\frac{1}{2} \sigma_{z,} \\
& \hat{\sigma}_{x}=\left(\begin{array}{ll}
0 & 1 \\
1 & 0
\end{array}\right), \hat{\sigma}_{y}=\left(\begin{array}{cc}
0 & -I \\
I & 0
\end{array}\right), \hat{\sigma}_{z}=\left(\begin{array}{cc}
1 & 0 \\
0 & -1
\end{array}\right),
\end{aligned}
$$

Where, Eqs (7b) are the Pauli Matrices [9].

If one substitutes Eqs (5,6,7a-b) into Eq (4) one finds after manipulations the following set of four first order differential equations in time for the four matrix elements of the defined Density Matrix. We note that the derived system of equations below were numerically verified versus $\mathrm{Eq}(9)$ below:

$$
\begin{aligned}
& \frac{d \rho_{11}[t]}{d t}=-\frac{I}{2} w_{1}[t]\left(\rho_{21}[t]-\rho_{12}[t]\right)+\mathrm{T}\left[\tau_{d p h}\right] \frac{1}{4}\left(w_{1}^{2}[t]\left(\rho_{22}[t]-\rho_{11}[t]\right)+w_{1}[t] \Delta w[t]\left(\rho_{21}[t]+\rho_{12}[t]\right)\right) \\
& \frac{d \rho_{22}[t]}{d t}=-\frac{d \rho_{11}[t]}{d t} \\
& \frac{d \rho_{12}[t]}{d t}=-\frac{I}{2}\left(2 \Delta w[t] \rho_{12}[t]+w_{1}[t]\left(\rho_{22}[t]-\rho_{11}[t]\right)+\mathrm{T}\left[\tau_{d p h}\right] \frac{1}{4}\left(w_{1}^{2}[t]\left(\rho_{21}[t]-\rho_{12}[t]\right)-2 \Delta^{2} w[t] \rho_{12}[t]+w_{1}[t] \Delta w[t]\left(\rho_{11}[t]-\rho_{22}[t]\right)\right)\right. \\
& \frac{d \rho_{21}[t]}{d t}=\frac{I}{2}\left(2 \Delta w[t] \rho_{21}[t]+w_{1}[t]\left(\rho_{22}[t]-\rho_{11}[t]\right)-\mathrm{T}\left[\tau_{d p h}\right] \frac{1}{4}\left(w_{1}^{2}[t]\left(\rho_{21}[t]-\rho_{12}[t]\right)+2 \Delta^{2} w[t] \rho_{12}[t]-w_{1}[t] \Delta w[t]\left(\rho_{11}[t]-\rho_{22}[t]\right)\right)\right.
\end{aligned}
$$

We note that for ease of manipulation and coding, one can rewrite Eqs (1) as: 


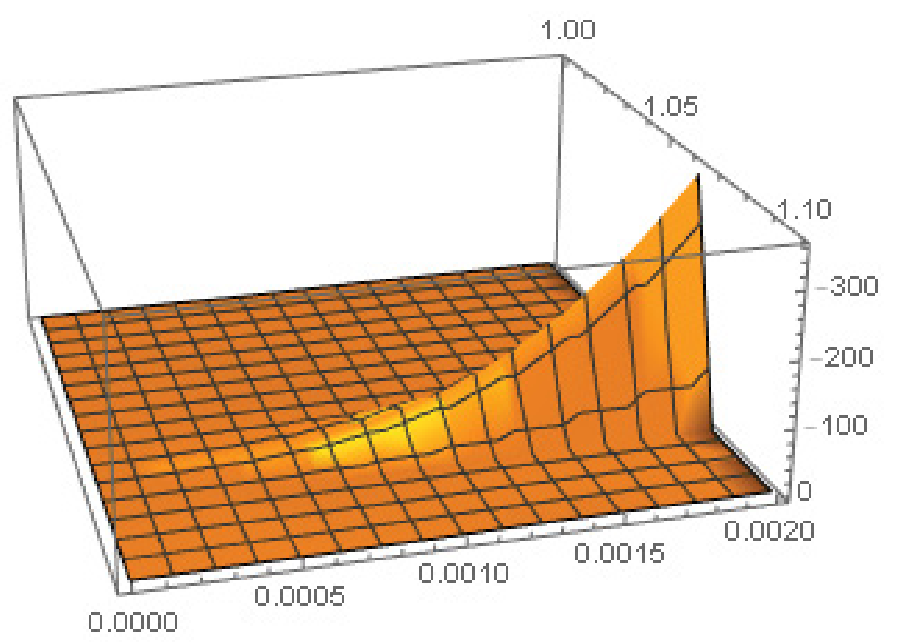

Figure 1a: $\sigma 11[t, \tau f \alpha]$.

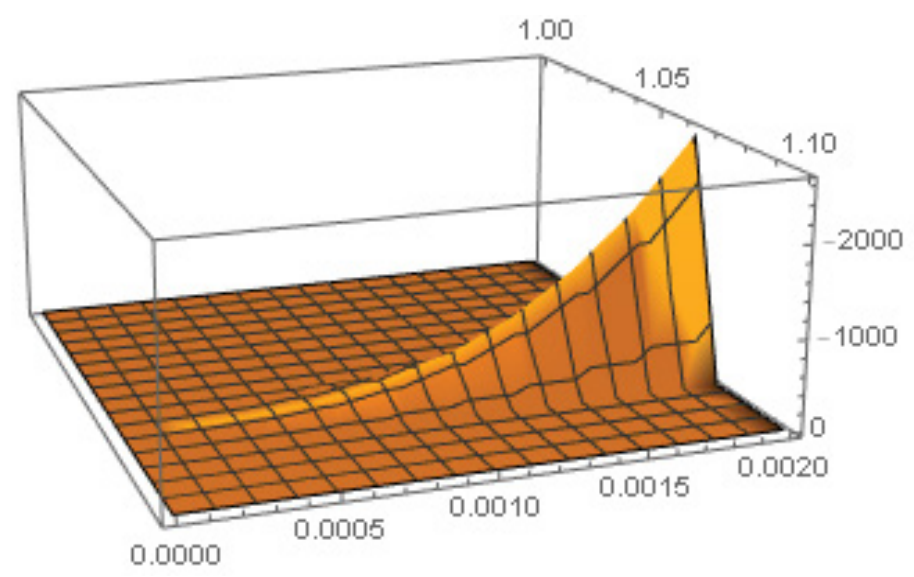

Figure 1b: $\sigma 12[t, \tau f \alpha]$.

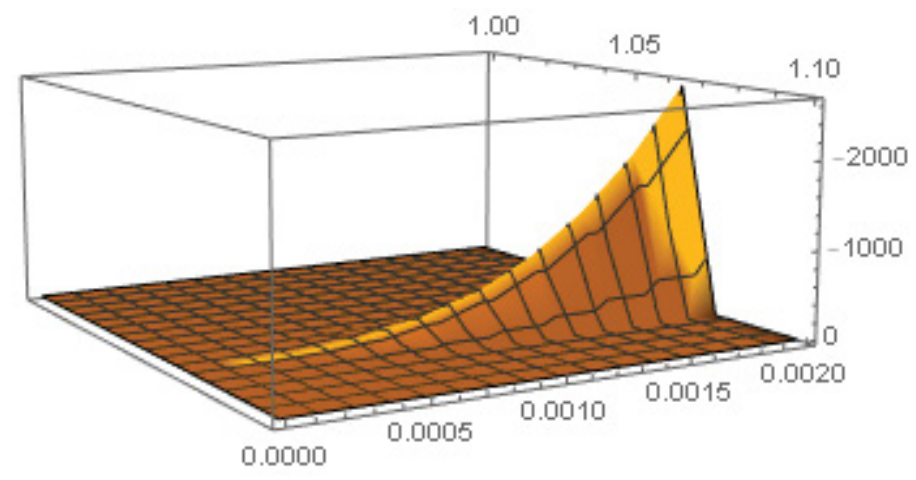

Figure 1c: $\sigma 21[t, \tau f \alpha]$.

$\frac{d \hat{\rho}[t]}{d t}=-I\left(\hat{H}_{R F}[t] \hat{\rho}[t]-\hat{\rho}[t] \hat{H}_{R F}[t]\right)+\mathrm{T}\left[\tau_{d p h}\right] \frac{1}{2}\left(2 \hat{H}_{R F}[t] \hat{\rho}[t] \hat{H}_{R F}[t]-\hat{H}_{R F}^{2}[t] \hat{\rho}[t]-\hat{\rho}[t] \hat{H}_{R F}^{2}[t]\right)$

Using a Numerical Platform such as Mathematica [11] one can readily Numerically Solve the four Differential equations Eqs $(8 \mathrm{a}, \mathrm{b}, \mathrm{c}, \mathrm{d})$ to obtain the Time-Dependent Matrix Elements $\rho_{i j}[t] ; i, j=1,2$. 


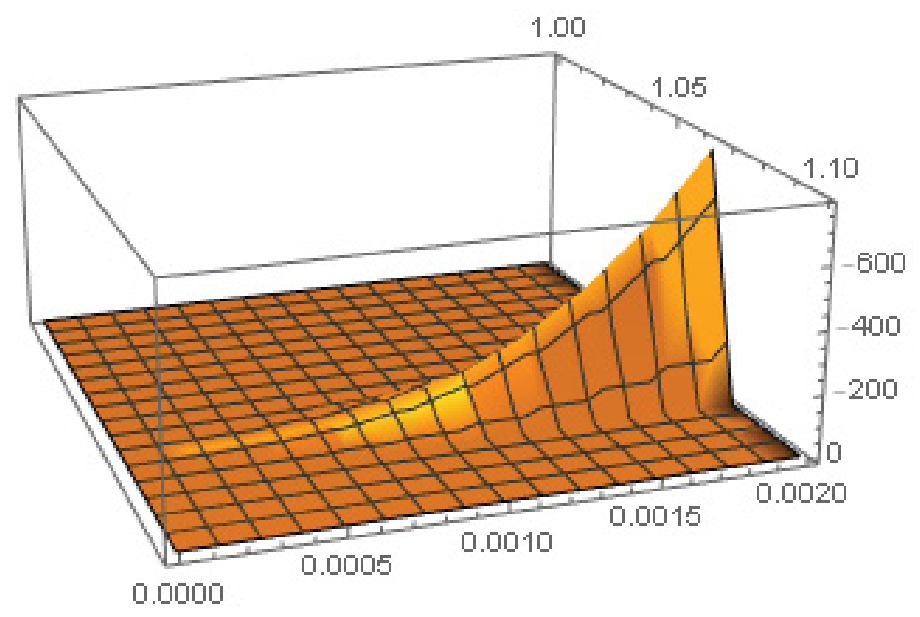

Figure 1d: $\sigma 22[t, \tau f \alpha]$.

\section{Results}

In Figure 1a, Figure $1 \mathrm{~b}$, Figure $1 \mathrm{c}$ and Figure $1 \mathrm{~d}$ we see plotted the $\left[t, \tau_{d p h}\right]$ dependence in ThreeDimensional Figures of the Four Density Matrix elements which are numerical solutions of Eqs [8a,b,c,d]. We note that the density matrix elements are considered dimensionless $[4,6,8,9]$. One can readily see there are Maxima at $\left[0.002 s, \tau_{f} 1.068\right] \tau_{f}=1.010^{-5} s$ over the domain considered.

In Summary, the Key Results of this effort, is the finding of Maxima for the dependence of Density Matrix element which indicate amplification of the coherences and populations for spin $1 / 2$ nuclei which are solutions of the system of Eqs $[8 \mathrm{a}, \mathrm{b}, \mathrm{c}, \mathrm{d}]$ that incorporate Decoherence effects at $\left[t, \tau_{d p h}\right]$. The system of Eqs [8] are to the knowledge of the author unique to the Magnetic Resonance literature.

Such Enhancement may prove to be of utility in Biomedical Applications in NMR/MRI, because the spin angular momenta can be expressed as sums and differences of the spin-1/2 Density Matrix elements which can be shown to exhibit pronounced amplification that are proportional to the Magnetization.

\section{References}

1. Kevin A, Gregg J (2009) Finite-time destruction of entanglement and non-locality by environmental influences. Foundations of Physics, arXiv:0903.0009.

2. Hornberger K (2009) Introduction to decoherence theory. In: Buchleitner A, Viviescas C, Tiersch M, Lecture Notes in Physics, Entanglement and Decoherence Berlin, Springer-Verlag 768: 221-276.

3. Heinz-Peter B, Francesco P (2010) The theory of open quantum systems. ( $4^{\text {th }}$ edn), Oxford University Press, New York, USA.

4. Maximilian S (2008) Decoherence. Quantum Physics, Springer-Verlag, New York, USA.

5. Michael AN, Isaac LC (2019) Quantum computation and quantum information. ( $8^{\text {th }}$ edn), Cambridge University Press, New York, USA.

6. Anatole A (1986) Principles of nuclear magnetism. Oxford University Press, New York, USA.

7. Brian C (1997) Nuclear magnetic resonance and relaxation. Cambridge University Press, New York, USA.

8. Maurice G (1988) Quantum description of high-resolution NMR in liquids. Oxford University Press, New York, USA.

9. Michael M (1988) Coherence and NMR. John Wiley and Sons, New York, USA.

10. Michael G, Lance De $L$ (2001) The return of the frequency sweep: Designing adiabatic pulses for contemporary NMR. J Magn Reson 153: 155-177.

11. Wolfram Research Mathematica, V12 Urbana, USA. 


\section{Appendix I:}

Definition of Adiabatic HS1 Pulse as defined by Garwood, et al. [10].

$w_{1}[t]=w_{1}^{M a x} \operatorname{Sech}\left[\beta\left(\frac{2 t}{t_{p}}-1\right)\right]$

$\Delta w[t]=\Omega_{A}-A_{\Lambda} \operatorname{Tanh}\left[\beta\left(\frac{2 t}{t_{p}}-1\right)\right]$

$w_{1}^{\operatorname{Max}}=\pi 10^{4} s^{-1}$

$\Omega_{A}=5000.0 s^{-1}$

$A_{\Lambda}=\pi 10^{4} \mathrm{~s}^{-1}$

tp is the Period of the Pulse.

$\beta=\operatorname{Arc} \operatorname{Tan}[0.01]$

\section{Appendix II:}

Outline of Derivation of Eqs [8 a,b,c,d].

We begin the development by restating Eq [4] of the Main Text:

$\frac{d \hat{\rho}[t]}{d t}=-I\left[\hat{H}_{R F}[t], \hat{\rho}[t]\right]+\mathrm{T}\left[\tau_{d p h}\right]\left(w_{1}^{2}[t] \hat{I}_{x} \hat{\rho}[t] \hat{I}_{x}+\Delta w^{2}[t] \hat{I}_{z} \hat{\rho}[t] \hat{I}_{z}\right.$

$\left.-\frac{\hat{1}}{4} w_{e f f}^{2}[t] \hat{\rho}[t]+w_{1}[t] \Delta w[t]\left(\hat{I}_{x} \hat{\rho}[t] \hat{I}_{z}+\hat{I}_{z} \hat{\rho}[t] \hat{I}_{x}\right)\right)$

We define the Following Terms:

$\hat{\rho}[t]=\left(\begin{array}{ll}\rho_{11}[t] & \rho_{12}[t] \\ \rho_{21}[t] & \rho_{22}[t]\end{array}\right)$

$\hat{I}_{x} \hat{\rho}[t] \hat{I}_{x}=\frac{1}{4}\left(\begin{array}{cc}\rho_{22}[t] & \rho_{21}[t] \\ \rho_{12}[t] & \rho_{11}[t]\end{array}\right)$

$\hat{I}_{z} \hat{\rho}[t] \hat{I}_{z}=\frac{1}{4}\left(\begin{array}{cc}\rho_{11}[t] & -\rho_{12}[t] \\ -\rho_{21}[t] & \rho_{22}[t]\end{array}\right)$

$\hat{I}_{x} \hat{\rho}[t] \hat{I}_{z}=\frac{1}{4}\left(\begin{array}{cc}\rho_{21}[t] & -\rho_{22}[t] \\ \rho_{11}[t] & -\rho_{12}[t]\end{array}\right)$

$\hat{I}_{z} \hat{\rho}[t] \hat{I}_{x}=\frac{1}{4}\left(\begin{array}{cc}\rho_{12}[t] & \rho_{11}[t] \\ -\rho_{22}[t] & -\rho_{21}[t]\end{array}\right)$

$\left[\hat{H}_{R F}[t], \hat{\rho}[t]\right]_{11}=\frac{1}{2} w_{1}[t]\left(\hat{\rho}_{21}[t]-\hat{\rho}_{12}[t]\right)$

$\left[\hat{H}_{R F}[t], \hat{\rho}[t]\right]_{22}=-\frac{1}{2} w_{1}[t]\left(\hat{\rho}_{21}[t]-\hat{\rho}_{12}[t]\right)$

All $(8 \mathrm{a}, \mathrm{b}, \mathrm{c}, \mathrm{d})$

$\left[\hat{H}_{R F}[t], \hat{\rho}[t]\right]_{12}=\frac{1}{2}\left(2 \Delta w[t] \hat{\rho}_{12}[t]+w_{1}[t]\left(\hat{\rho}_{22}[t]-\hat{\rho}_{11}[t]\right)\right)$

$\left[\hat{H}_{R F}[t], \hat{\rho}[t]\right]_{21}=-\left[\hat{H}_{R F}[t], \hat{\rho}[t]\right]_{12}$

If one substitutes Eqs All ( 2-8) into Eq All(1) one obtains Eqs (8 a,b,c,d). 